\title{
Analytic Tableau Calculi for KLM Rational Logic R
}

\author{
Laura Giordano $^{1}$, Valentina Gliozzi ${ }^{2}$, Nicola Olivetti $^{3}$, and Gian Luca Pozzato ${ }^{2}$ \\ 1 Dipartimento di Informatica - Università del Piemonte Orientale A. Avogadro - via Bellini \\ 25/G - 15100 Alessandria, Italy - e-mail: laura@mfn. unipmn. it \\ 2 Dipartimento di Informatica - Università degli Studi di Torino, corso Svizzera 185 - 10149 \\ Turin - Italy - e-mail: $\{$ gliozzi, pozzato\}@di.unito.it \\ 3 LSIS - UMR CNRS 6168 Université Paul Cézanne (Aix-Marseille 3) Avenue Escadrille \\ Normandie-Niemen 13397 Marseille Cedex 20 - France - e-mail: \\ nicola.olivetti@univ.u-3mrs.fr - nicola.olivetti@lsis.org
}

\begin{abstract}
In this paper we present a tableau calculus for the rational logic $\mathbf{R}$ of default reasoning, introduced by Kraus, Lehmann and Magidor. Our calculus is obtained by introducing suitable modalities to interpret conditional assertions, and makes use of labels to represent possible worlds. We also provide a decision procedure for $\mathbf{R}$, and study its complexity.
\end{abstract}

\section{Introduction}

In [1] Kraus, Lehmann and Magidor (KLM) proposed a formalization of nonmonotonic reasoning that led to a classification of nonmonotonic consequence relations, determining a hierarchy of stronger and stronger systems. The so called KLM properties have been widely accepted as the "conservative core" of default reasoning. The role of KLM logics is similar to the role of AGM postulates in Belief Revision [2]: they give a set of postulates for default reasoning that any concrete reasoning mechanism should satisfy.

In the recent literature it is shown that many different approaches to default reasoning are characterized by these properties. In particular, a recent work by Halpern and Friedman [3] has shown that two of these systems, namely preferential $\operatorname{logic} \mathbf{P}$ and rational logic $\mathbf{R}$, are natural and general systems: surprisingly enough, the axiom systems of these logics are complete with respect to a wide spectrum of semantics (including $\kappa$-rankings, parametrized probabilistic structures, $\epsilon$-semantics and possibilistic structures). The reason is that all these structures are examples of plausibility structures and the truth in them is captured by the axioms of preferential or rational logic.

The results presented in [3], and their extensions to the first order setting [4], are the source of a renewed interest in the KLM framework. A considerable amount of research in the area has then concentrated in developing concrete mechanisms for plausible reasoning in accordance with KLM systems (P and $\mathbf{R}$ mostly). These mechanisms are defined by exploiting a variety of models of reasoning under uncertainty (ranked models, belief functions, possibilistic logic, etc. [5-10]) that provide, as we remarked, alternative semantics to KLM systems. The mechanisms can be seen as restricting the consideration to preferred classes of models of KLM logics; this is also the case of Lehmann's notion of rational closure (not to be confused with the logic R). More recent research has also explored the integration of KLM framework with paraconsistent logics [11]. Finally, there has been some recent investigation on the relation between KLM systems and decision-theory $[12,13]$. 
In KLM logics, defeasible knowledge is assumed to be represented by a set of nonmonotonic conditionals or assertions of the form $A \sim B$, whose reading is normally (or typically) the A's are B's. The operator " $\sim$ " is nonmonotonic, in the sense that $A \sim B$ does not imply $A \wedge C \sim B$.

In this paper we focus on logic $\mathbf{R}$, whose axiom system includes the rule of rational monotonicity: if $A \sim B$ and $\neg(A \sim \neg C)$ hold, then one can infer $A \wedge C \sim B$. This rule allows a conditional to be inferred from a set of conditionals in absence of other information. More precisely, "it says that an agent should not have to retract any previous defeasible conclusion when learning about a new fact the negation of which was not previously derivable" [14].

Consider, for instance, a knowledge base $K$ containing the following set of conditional assertions: adult $\sim$ worker, adult $\sim$ taxpayer, student $\sim$ adult, student $\sim$ $\neg$ worker, student $\sim \neg$ taxpayer, whose meaning is that adults typically work, adults typically pay taxes, students are typically adults, but they typically do not work, nor do they pay taxes. In rational logic $\mathbf{R}$ one can infer the following conditional assertions from the knowledge base $K$ : adult $\sim \neg$ student (i.e. typical adults are not students), adult $\wedge$ student $\sim \neg$ worker (giving preference to more specific information). Moreover, if one further knows that $\neg$ (adult $\sim \neg$ married) (i.e. it is not the case the adults are typically unmarried), one can also infer that adult $\wedge$ married $\sim$ worker. Observe that one cannot infer student $\sim$ worker.

From a semantic point of view, the models of rational logic are possible-world structures equipped with a preference relation among worlds. The preference relation (an irreflexive and transitive relation) on worlds is further assumed to be modular. The meaning of a conditional assertion $A \sim B$ is that $B$ holds in the most preferred worlds where $A$ holds.

In this work we extend the investigation of tableau procedures for propositional KLM logics developed in [15] by considering the case of $\mathbf{R}$. Our approach is based on a novel interpretation of $\mathbf{R}$ into modal logics. As a difference with previous approaches (e.g. Crocco et. al [16] and Boutillier [17]), that take S4.3 as the modal counterpart of $\mathbf{R}$, we consider here an extension of modal logic $\mathrm{G}$, including modularity of the preference relation. The idea is simply to interpret the preference relation as an accessibility relation: a conditional $A \sim B$ holds in a model if $B$ is true in all minimal $A$-worlds, i.e. worlds in which $A$ holds, that are minimal w.r.t. $<$. A world is $A$-minimal if all smaller worlds are not $A$-worlds. The relation with modal logic $\mathrm{G}$ is motivated by the fact that we assume, following KLM, the so-called smoothness condition, which is related to the well-known limit assumption. This condition ensures that minimal $A$-worlds exist, by preventing infinitely descending chains of worlds. This condition is therefore ensured by the finite-chain condition on the accessibility relation (as in modal logic G). As it has been done in [15] for preferential logic and loop-cumulative logic, our tableau method provides a sort of run-time translation of $\mathbf{R}$ into the extension of modal logic $\mathrm{G}$. As a difference with [15], we develop here a labelled tableau system, which seems to be the most natural approach.

The paper presents a tableau calculus for $\mathbf{R}$ which is sound, complete and terminating. Moreover, it defines a systematic procedure which allows the satisfiability problem 
for $\mathbf{R}$ to be decided in nondeterministic polynomial time, in accordance with the known complexity results for this logic.

\section{KLM Rational Logic R}

In this section we briefly recall the axiomatization and semantics of the rational logic R. For a complete description of KLM systems, see [1] and [14]. The language of KLM logics consists just of conditional assertions $A \sim B$. We consider a richer language allowing boolean combinations of conditional and propositional formulas ${ }^{1}$. Our language $\mathcal{L}$ is defined from a set of propositional variables $A T M$, the boolean connectives and the conditional operator $\sim$. We use $A, B, C, \ldots$ to denote propositional formulas, whereas $F, G, \ldots$ are used to denote all formulas (even conditionals); $\Gamma, \Delta, \ldots$ represent sets of formulas. The formulas of $\mathcal{L}$ are defined as follows: if $A$ is a propositional formula, $A \in \mathcal{L}$; if $A$ and $B$ are propositional formulas, $A \sim B \in \mathcal{L}$; if $F$ is a boolean combination of formulas of $\mathcal{L}, F \in \mathcal{L}$. $\mathcal{L}$ corresponds to the fragment of the language of conditional logics without nested conditionals $\sim$.

The axiomatization of $\mathbf{R}$ consists of all axioms and rules of propositional calculus together with the following axioms and rules (notice that $\vdash$ denotes provability in the propositional calculus):

- REF. $A \sim A$ (reflexivity)

- LLE. If $\vdash A \leftrightarrow B$, then $\vdash(A \sim C) \rightarrow(B \sim C)$ (left logical equivalence)

- RW. If $\vdash A \rightarrow B$, then $\vdash(C \sim A) \rightarrow(C \sim B)$ (right weakening)

- AND. $((A \sim B) \wedge(A \sim C)) \rightarrow(A \sim B \wedge C)$

- OR. $((A \sim C) \wedge(B \sim C)) \rightarrow(A \vee B \sim C)$

- CM. $((A \sim B) \wedge(A \sim C)) \rightarrow(A \wedge B \sim C)$ (cautious monotonicity)

- RM. $((A \sim B) \wedge \neg(A \sim \neg C)) \rightarrow(A \wedge C \sim B)$ (rational monotonicity)

REF states that $A$ is always a default conclusion of $A$. LLE states that the syntactic form of the antecedent of a conditional formula is irrelevant. RW describes a similar property of the consequent. This allows to combine default and logical reasoning [3]. AND states that it is possible to combine two default conclusions. OR states that it is allowed to reason by cases: if $C$ is the default conclusion of two premises $A$ and $B$, then it is also the default conclusion of their disjunction. CM states that if $B$ and $C$ are two default conclusions of $A$, then adding one of the two conclusions to $A$ will not cause the retraction of the other conclusion. As explained in the Introduction, RM captures a natural form of monotonicity.

The semantics of $\mathbf{R}$ is defined by considering possible world structures with a preference relation (a strict partial order) $w<w^{\prime}$ whose meaning is that $w$ is preferred to $w^{\prime}$. Moreover, the preference relation is supposed to be modular, i.e. for all $w, w_{1}$ and $w_{2}$, if $w_{1}<w_{2}$ then either $w_{1}<w$ or $w<w_{2}$. We have that $A \sim B$ holds in a model $\mathcal{M}$ if $B$ holds in all minimal $A$-worlds (w.r.t. $<$ ). This definition makes sense provided minimal $A$-worlds exist (whenever there are $A$-worlds). This is ensured by the smoothness condition in the next definition.

\footnotetext{
${ }^{1}$ In [14] it is shown that in a language lacking boolean combinations of conditionals, system $\mathbf{R}$ collapses into system $\mathbf{P}$. As shown in [3], the two systems are distinct given a richer language (as ours) allowing boolean combinations of conditionals.
} 
Definition 1 (Semantics of R, Definition 14 in [14]). A rational model is a triple $\mathcal{M}=$ $\langle\mathcal{W},<, V\rangle$ where: $\mathcal{W}$ is a non-empty set of items called worlds; $<$ is an irreflexive, transitive and modular relation on $\mathcal{W} ; V$ is a function $V: \mathcal{W} \longmapsto$ pow $(A T M)$, which assigns to every world $w$ the set of atoms holding in that world. We define the truth conditions for a formula $F$ as follows:

- If $F$ is a boolean combination of formulas, $\mathcal{M}, w=F$ is defined as for propositional logic;

- Let $A$ be a propositional formula; we define $\operatorname{Min}_{<}(A)=\{w \in \mathcal{W}|\mathcal{M}, w|=A$ and $\forall w^{\prime}, w^{\prime}<w$ implies $\left.\mathcal{M}, w^{\prime} \not \models A\right\}$;

- $\mathcal{M}, w \models A \sim B$ iffor all $w^{\prime}$, if $w^{\prime} \in \operatorname{Min}_{<}(A)$ then $\mathcal{M}, w^{\prime} \models B$.

The relation $<$ satisfies the smoothness condition: if $\mathcal{M}, w \models A$ then $w \in \operatorname{Min}_{<}(A)$ or $\exists w^{\prime} \in \operatorname{Min}_{<}(A)$ such that $w^{\prime}<w$.

We say that a formula $F$ is valid in a model $\mathcal{M}(\mathcal{M} \models F)$, if $\mathcal{M}, w \models F$ for every $w \in \mathcal{W}$. A formula is valid if it is valid in every model $\mathcal{M}$.

Notice that the truth conditions for conditional formulas are given with respect to single possible worlds for uniformity sake. Since the truth value of a conditional only depends on global properties of $\mathcal{M}$, we have that: $\mathcal{M}, w \models A \sim B$ iff $\mathcal{M} \models A \sim B$.

By transitivity of the relation $<$, the smoothness condition is equivalent to the following strong smoothness condition: given a formula $A$ and a world $w$, if there is $w^{\prime}<w$ such that $\mathcal{M}, w^{\prime} \models A$, then either $w^{\prime} \in \operatorname{Min}_{<}(A)$ or there exists $w^{\prime \prime}<w$ such that $w^{\prime \prime} \in \operatorname{Min}_{<}(A)$. Observe also that by the modularity of $<$ it follows that possible worlds of $\mathcal{W}$ are clustered into equivalence classes, each class consisting of worlds that are incomparable to one another; the classes are totally ordered ${ }^{2}$. In other words the property of modularity determines a ranking of worlds so that the semantics of $\mathbf{R}$ can be specified equivalently in terms of ranked models [14]. By means of the modularity condition on the preference relation, we can also prove the following theorem:

Theorem 1 (Small Model Theorem). For any $\Gamma \subseteq \mathcal{L}$, if $\Gamma$ is satisfiable in a rational model, then it is satisfiable in a rational model containing at most $n$ worlds, where $n$ is the size of $\Gamma$, i.e. the length of the string representing $\Gamma$.

\section{The Tableau Calculus for $\mathbf{R}$}

In this section we present $\mathcal{T} \mathbf{R}$, a labelled tableau calculus for rational logic $\mathbf{R}$. The calculus makes use of labels to represent possible worlds. We consider a language $\mathcal{L}_{R}$ and a denumerable alphabet of labels $\mathcal{A}$, whose elements are denoted by $x, y, z, \ldots$. $\mathcal{L}_{R}$ extends $\mathcal{L}$ by formulas of the form $\square A$, where $A$ is propositional, whose intuitive meaning is as follows: $\square A$ holds in a world $w$ if $A$ holds in all the worlds $w^{\prime}$ such that $w^{\prime}<w$, that is to say:

Definition 2 (Truth condition of $\square$ ). $\mathcal{M}, w \models \square A$ if for every $w^{\prime} \in \mathcal{W}$ if $w^{\prime}<w$ then $\mathcal{M}, w^{\prime} \models A$.

\footnotetext{
${ }^{2}$ Notice that the worlds themselves may be incomparable since the relation $<$ is not assumed to be (weakly) connected.
} 
It is easy to see that $\square$ has (among others) the properties of the modal system G, whose characterizing axiom is $\square(\square A \rightarrow A) \rightarrow \square A$ (see for instance [18]). This axiom guarantees that the accessibility relation (defined as $x R y$ if $y<x$ ) is transitive and does not have infinite ascending chains. From definition of $\operatorname{Min}_{<}(A)$ in Definition 1 above, it follows that for any formula $A, w \in \operatorname{Min}_{<}(A)$ iff $\mathcal{M}, w \models A \wedge \square \neg A$. As we will see, $\mathcal{T} \mathbf{R}$ will only make use of boxed formulas with a negated argument, i.e. with the form $x: \square \neg A$.

Our tableau calculus comprises two kinds of labelled formulas: $(i)$ world formulas $x: F$, whose meaning is that $F$ holds in the possible world represented by $x ;(i i)$ relation formulas of the form $x<y$, where $x, y \in \mathcal{A}$, used to represent the relation $<$. We denote by $\alpha, \beta \ldots$ a world or a relation formula.

We define $\Gamma_{x \rightarrow y}^{M}=\{y: \neg A, y: \square \neg A \mid x: \square \neg A \in \Gamma\}$. The calculus $\mathcal{T} \mathbf{R}$ is presented in Figure 1. We call dynamic the rules $\left(\sim^{-}\right)$and $\left(\square^{-}\right)$that introduce new labels in their conclusion; all the other rules are called static.

$$
\begin{aligned}
& \text { (AX) } \Gamma, x: P, x: \neg P \quad \text { with } P \in A T M \\
& (\neg) \frac{\Gamma, x: \neg \neg F}{\Gamma, x: F} \\
& \left(\wedge^{+}\right) \frac{\Gamma, x: F \wedge G}{\Gamma, x: F, x: G} \\
& \left(\wedge^{-}\right) \frac{\Gamma, x: \neg(F \wedge G)}{\Gamma, x: \neg F \quad \Gamma, x: \neg G} \\
& \left(\sim^{+}\right) \frac{\Gamma, u: A \sim B}{\Gamma, x: \neg A, u: A \sim B \quad \Gamma, x: \neg \square \neg A, u: A \sim B \quad \Gamma, x: B, u: A \sim B} \\
& \left(\sim^{-}\right) \frac{\Gamma, u: \neg(A \sim B)}{\Gamma, x: A, x: \square \neg A, x: \neg B} x \text { new label } \quad\left(\square^{-}\right) \frac{\Gamma, x: \neg \square \neg A}{\Gamma, y<x, \Gamma_{x \rightarrow y}^{M}, y: A, y: \square \neg A} y \text { new label } \\
& (<) \frac{\Gamma, x<y}{\Gamma, x<y, z<y, \Gamma_{y \rightarrow z}^{M} \quad \Gamma, x<y, x<z, \Gamma_{z \rightarrow x}^{M}} z \text { occurs in } \Gamma \text { and }\{x<z, z<y\} \cap \Gamma=\emptyset
\end{aligned}
$$

Fig. 1. The calculus $\mathcal{T} R$. To save space, rules for $\rightarrow$ and $\vee$ are omitted.

Definition 3 (Truth conditions of formulas of $\mathcal{T} \mathbf{R}$ ). Given a model $\mathcal{M}=\langle\mathcal{W},<, V\rangle$ and a labelled alphabet $\mathcal{A}$, we consider a mapping $I: \mathcal{A} \mapsto \mathcal{W}$. Given a formula $\alpha$ of the calculus $\mathcal{T} \mathbf{R}$, we define $\mathcal{M} \models_{I} \alpha$ as follows: $\mathcal{M} \models_{I} x: F$ iff $\mathcal{M}, I(x) \models F$; $\mathcal{M} \models_{I} x<y$ iff $I(x)<I(y)$.

We say that a set $\Gamma$ of formulas of $\mathcal{T} \mathbf{R}$ is satisfiable if, for all formulas $\alpha \in \Gamma$, we have that $\mathcal{M} \models_{I} \alpha$, for some model $\mathcal{M}$ and some mapping $I$. 
A tableau is a tree whose nodes are sets of formulas $\Gamma$. Therefore, a branch is a sequence of sets of formulas $\Gamma_{1}, \Gamma_{2}, \ldots, \Gamma_{n}, \ldots$ Each node $\Gamma_{i}$ is obtained by its immediate predecessor $\Gamma_{i-1}$ by applying a rule of $\mathcal{T} \mathbf{R}$, having $\Gamma_{i-1}$ as the premise and $\Gamma_{i}$ as one of its conclusions. A branch is closed if one of its nodes is an instance of $(\mathbf{A X})$, otherwise it is open. We say that a tableau is closed if all its branches are closed.

In order to verify that a set of formulas $\Gamma$ is unsatisfiable, we label all the formulas in $\Gamma$ with a new label $x$, and verify that the resulting set of labelled formulas has a closed tableau. For instance, in order to verify that the set $\{$ adult $\sim$ worker, $\neg$ (adult $\sim$ $\neg$ married), $\neg$ (adult $\wedge$ married $\sim$ worker $)\}$ is unsatisfiable (thus adult $\wedge$ married $\sim$ worker is entailed by $\{$ adult $\sim$ worker, $\neg($ adult $\sim \neg$ married $)\}$ ), we can build the closed tableau in Figure 2.

$$
\begin{aligned}
& x: a \sim w, x: \neg(a \sim \neg m), x: \neg(a \wedge m \sim w) \\
& x: a \sim w, y: a, y: \square \neg a, y: \neg \neg m, x: \neg(a \wedge m \sim w)(\sim \\
& x: a \sim w, y: a, y: \square \neg a, y: m, x: \neg(a \wedge m \sim w) \\
& x: a \sim w, y: a, y: \square \neg a, y: m, z: a \wedge m, z: \square \neg(a \wedge m), z: \neg w)\left(\sim^{-}\right) \\
& \left.\ldots, y: \neg a, y: a \quad x: a \sim w, y: w, y: a, y: \square \neg a, y: m, z: a \wedge m, z: \square \neg(a \wedge m), z: \neg w{ }^{+}\right) \quad \ldots, y: \neg \square \neg a, y: \square \neg a\left(\sim^{+}\right)
\end{aligned}
$$

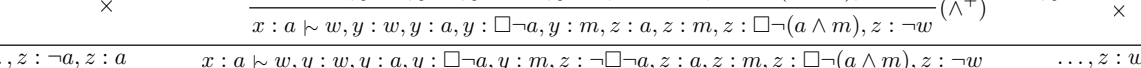

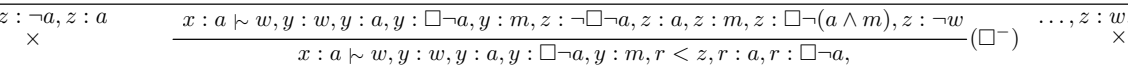

$$
\begin{aligned}
& r: \neg(a \wedge m), r: \square \neg(a \wedge m), z: a, z: m, z: \square \neg(a \wedge m), z: \neg w
\end{aligned}
$$

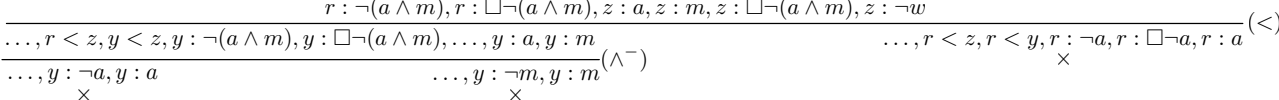

Fig. 2. A derivation in $\mathcal{T} \mathbf{R}$ of \{adult $\sim$ worker, $\neg$ (adult $\sim \neg$ married $), \neg($ adult $\wedge$ married $\sim$ worker $)\}$. To save space, we use $a$ for adult, $m$ for married, and $w$ for worker.

Lemma 1. For any set of formulas $\Gamma$ and any world formula $x: F$, there is a closed tableau for $\Gamma, x: F, x: \neg F$.

The calculus $\mathcal{T} \mathbf{R}$ is sound and complete w.r.t. the semantics.

Theorem 2 (Soundness). If there is a closed tableau for a set of formulas $\Gamma$, then $\Gamma$ is unsatisfiable.

Proof. By induction on the height of the closed tableau for $\Gamma$. If $\Gamma$ is an axiom, then $x: P \in \Gamma$ and $x: \neg P \in \Gamma$, therefore there is no $w \in \mathcal{W}$ such that $\mathcal{M}, w \models P$ and $\mathcal{M}, w \not \forall P$, and $\Gamma$ is unsatisfiable. For the inductive step, we prove the contrapositive, i.e. we prove for each rule that, if the premise is satisfiable, so is (at least) one of the conclusions. To save space, we only present the most interesting case of $\left(\square^{-}\right)$. Since the premise is satisfiable, then there is a model $\mathcal{M}$ and a mapping $I$ such that $\mathcal{M} \models_{I} \Gamma, x: \neg \square \neg A$. Let $w \in \mathcal{W}$ such that $I(x)=w$; this means that $\mathcal{M}, w \not \models \square \neg A$, hence there exists a world $w^{\prime}<w$ such that $\mathcal{M}, w^{\prime} \models A$. By the strong smoothness condition, we have that there exists a minimal such world, so we can assume that $w^{\prime} \in \operatorname{Min}_{<}(A)$, thus $\mathcal{M}, w^{\prime} \models \square \neg A$. In order to prove that the conclusion of the rule is satisfiable, we construct a mapping $I^{\prime}$ as follows: let $y$ be a new label, not occurring 
in the current branch; we define (1) $I^{\prime}(u)=I(u)$ for all $u \neq y$ and $(2) I^{\prime}(y)=w^{\prime}$. Since $y$ does not occur in $\Gamma$, it follows that $\mathcal{M} \models_{I^{\prime}} \Gamma$. By Definition 3, we have that $\mathcal{M}=_{I^{\prime}} y<x$ since $w^{\prime}<w$. Moreover, since $I^{\prime}(y)=w^{\prime}$, we have that $\mathcal{M}=_{I^{\prime}} y: A$ and $\mathcal{M} \models_{I^{\prime}} y: \square \neg A$. Finally, $\mathcal{M} \models_{I^{\prime}} \Gamma_{x \rightarrow y}^{M}$ follows from the fact that $I^{\prime}(y)<I^{\prime}(x)$ and from the transitivity of $<$. The only conclusion of the rule is then satisfiable in $\mathcal{M}$ via $I^{\prime}$.

In order to prove the completeness of the calculus, we introduce the notion of saturated branch and we show that $\mathcal{T} \mathbf{R}$ introduces a finite number of labels in a tableau.

Definition 4 (Saturated branch). We say that a branch $\mathbf{B}=\Gamma_{1}, \Gamma_{2}, \ldots, \Gamma_{n}, \ldots$ of a tableau is saturated if the following conditions hold: (1) For the boolean connectives, the condition of saturation is defined in the usual way. For instance, if $x: A \wedge B \in \Gamma_{i}$ in $\mathbf{B}$, then there exists $\Gamma_{j}$ in $\mathbf{B}$ such that $x: A \in \Gamma_{j}$ and $x: B \in \Gamma_{j}$. (2) If $x: A \sim$ $B \in \Gamma_{i}$, then for any label $y$ in $\mathbf{B}$, there exists $\Gamma_{j}$ in $\mathbf{B}$ such that either $y: \neg A \in \Gamma_{j}$ or $y: \neg \square \neg A \in \Gamma_{j}$ or $y: B \in \Gamma_{j}$. (3) If $x: \neg(A \sim B) \in \Gamma_{i}$, then there is a $\Gamma_{j}$ in $\mathbf{B}$ such that, for some $y, y: A \in \Gamma_{j}, y: \square \neg A \in \Gamma_{j}$, and $y: \neg B \in \Gamma_{j}$. (4) If $x: \neg \square \neg A \in \Gamma_{i}$, then there exists $\Gamma_{j}$ in $\mathbf{B}$ such that, for some $y, y<x \in \Gamma_{j}$, $y: A \in \Gamma_{j}$ and $y: \square \neg A \in \Gamma_{j}$. (5) If $x<y \in \Gamma_{i}$, then for all labels $z$ in $\mathbf{B}$, there exists $\Gamma_{j}$ in $\mathbf{B}$ such that either $z<y \in \Gamma_{j}$ or $x<z \in \Gamma_{j}$.

We can easily show the following Lemma:

Lemma 2. Given a tableau starting with $x_{0}: F$, for any saturated branch $\mathbf{B}=$ $\Gamma_{1}, \Gamma_{2}, \ldots, \Gamma_{n}, \ldots$, we have that:

- if $z<y \in \Gamma_{i}$ in $\mathbf{B}$ and $y<x \in \Gamma_{j}$ in $\mathbf{B}$, then there exists $\Gamma_{k}$ in $\mathbf{B}$ such that $z<x \in \Gamma_{k}$

- if $x: \square \neg A \in \Gamma_{i}$ in $\mathbf{B}$ and $y<x \in \Gamma_{j}$ in $\mathbf{B}$, then there exists $\Gamma_{k}$ in $\mathbf{B}$ such that $y: \neg A \in \Gamma_{k}$ and $y: \square \neg A \in \Gamma_{k}$;

- for no $\Gamma_{i}$ in $\mathbf{B}, x<x \in \Gamma_{i}$.

Notice that in $\mathcal{T} \mathbf{R}$ the order of application of the rules is not relevant, since all the rules are invertible. Hence, no backtracking is required in the calculus, and we can assume without loss of generality that a given set of formulas $\Gamma$ has a unique tableau.

In Theorem 3 below we prove that the tableau for a given set of formulas $\Gamma_{0}$ contains a finite number of labels. Indeed, the only rules that can introduce new labels in the tableau are $\left(\sim^{-}\right)$and $\left(\square^{-}\right)$. We prove that in the tableau there can be only finitely many applications of these rules. Intuitively, the rule $\left(\sim^{-}\right)$can be applied only once for each negated conditional $\Gamma$ (hence it introduces only a finite number of labels). Furthermore, the generation of infinite branches due to the interplay between rules $\left(\sim^{+}\right)$and $\left(\square^{-}\right)$ cannot occur. Indeed, each application of $\left(\square^{-}\right)$to a formula $x: \neg \square \neg A$ (introduced by $\left(\sim^{+}\right)$) adds the formula $y: \square \neg A$ to the conclusion, so that $\left(\sim^{+}\right)$can no longer consistently introduce $y: \neg \square \neg A$. This is due to the properties of $\square$, that are similar to the corresponding modality of modal system $\mathrm{G}$.

In order to prove this result in a rigorous manner, we proceed as follows: first, we introduce the measure of Definition 6, and the auxiliary Definition 5; then, we prove 
that each application of $\left(\sim^{-}\right)$and $\left(\square^{-}\right)$reduces this measure, until the two rules are no longer applicable. We write $A \sim B \epsilon_{+} \Gamma$ (resp. $A \sim B \epsilon_{-} \Gamma$ ) if $A \sim B$ occurs positively (resp. negatively) in $\Gamma$, where positive and negative occurrences are defined in the standard way.

Definition 5. Given an initial set of formulas $\Gamma_{0}$, we define: $(i)$ the set $\mathcal{L}_{\square+}^{\Gamma_{0}}$ of boxed formulas $\square \neg A$ that can be generated in a tableau for $\Gamma_{0}$, i.e. $\mathcal{L}_{\square^{+}}^{\Gamma_{0}}=\{\square \neg A \mid A \sim$ $\left.B \in_{+} \Gamma_{0}\right\} \cup\left\{\square \neg A \mid A \sim B \in \in_{-} \Gamma_{0}\right\}$. We let $n_{0}=\left|\mathcal{L}_{\square^{+}}^{\Gamma_{0}}\right| ;($ ii $)$ the multiset $\mathcal{L}_{\square^{-}}^{\Gamma_{0}}$ of negated boxed formulas that can be generated in a tableau for $\Gamma_{0}$, i.e. $\mathcal{L}_{\square^{-}}^{\Gamma_{0}}=[\neg \square \neg A \mid$ $\left.A \sim B \in_{+} \Gamma_{0}\right]$. We let $k_{0}=\left|\mathcal{L}_{\square^{-}}^{\Gamma_{0}}\right|$.

Given a label $x$ and a set of formulas $\Gamma$ in the tableau for the initial set $\Gamma_{0}$, we define: (i) the number $n_{x}$ of positive boxed formulas $\square \neg A$ not labelled by $x$, i.e. $n_{x}=$ $n_{0}-\left|\left\{\square \neg A \in \mathcal{L}_{\square+}^{\Gamma_{0}} \mid x: \square \neg A \in \Gamma\right\}\right|$; (ii) the number $k_{x}$ of negated boxed formulas $\neg \square \neg A$ not yet expanded in a world $x$, i.e. $k_{x}=k_{0}-\mid\left[\neg \square \neg A \in \mathcal{L}_{\square^{-}}^{\Gamma_{0}} \mid x\right.$ : $\neg \square \neg A$ has been expanded $]$.

Definition 6. We define $p(\Gamma)=\left\langle c_{1}, c_{2}\right\rangle$ where:

- $c_{1}=\left|\left\{u: A \sim B \in \epsilon_{-} \Gamma\right\}\right|$

- $c_{2}$ is the multiset given by $\left[c_{2}^{x_{1}}, c_{2}^{x_{2}}, \ldots, c_{2}^{x_{n}}\right]$, where $x_{1}, x_{2}, \ldots, x_{n}$ are the labels occurring in $\Gamma$ and, given a label $x, c_{2}^{x}$ is a pair $\left(n_{x}, k_{x}\right)$ in a lexicographic order ( $n_{x}$ and $k_{x}$ are defined as in Definition 5). We consider the integer multiset ordering given by $c_{2}$.

We consider the lexicographic order given by $p(\Gamma)$.

Roughly speaking, $c_{1}$ is the number of negated conditionals that can still be expanded in the tableau. $c_{2}$ keeps track of positive conditionals which can still create $a$ new world. The application of $\left(\sim^{-}\right)$reduces $c_{1}$. The application of $\left(\square^{-}\right)$reduces $c_{2}$. Indeed, if $\left(\sim^{+}\right)$is applied to $u: A \sim B$, this application introduces a branch containing $x: \neg \square \neg A$; when a new world $y$ is generated by an application of $\left(\square^{-}\right)$on $x: \neg \square \neg A$, $y: \square \neg A$ is added to the current set of formulas. If $\left(\sim^{+}\right)$is applied to $u: A \sim B$ by using the new world $y$, then the conclusion where $y: \neg \square \neg A$ is introduced is closed, by the presence of $y: \square \neg A$.

Theorem 3. Given a set of formulas $\Gamma$, the tableau generated by $\mathcal{T} \mathbf{R}$ for $\Gamma$ only contains a finite number of labels.

Proof sketch. First, we can easily prove that each application of $\left(\sim^{-}\right)$and $\left(\square^{-}\right)$reduces $p(\Gamma)$. This means that a finite number of applications of these rules leads either to a node containing $x: F, x: \neg F$ (see Lemma 1) or to a node to which the two rules are no further applicable. In particular, when $c_{1}=0,\left(\sim^{-}\right)$is no longer applicable. When $c_{2}=[(0,0),(0,0), \ldots,(0,0)]$, we can reason as follows: suppose there is $x: \neg \square \neg A \in \Gamma$; since $c_{2}^{x}=(0,0)$, it follows that $x: \square \neg A \in \Gamma$, and we conclude by Lemma 1. 
Theorem 4 (Completeness). If a set of formulas $\Gamma$ is unsatisfiable, then it has a closed tableau.

Proof sketch. We show the contrapositive, i.e. if there is no closed tableau for $\Gamma$, then $\Gamma$ is satisfiable. Consider the tableau starting with the set of formulas $\{x: F$ such that $F \in \Gamma\}$ and any open, saturated branch $\mathbf{B}=\Gamma_{1}, \Gamma_{2}, \ldots, \Gamma_{n}$ in it. Starting from $\mathbf{B}$, we build a canonical model $\mathcal{M}=\left\langle\mathcal{W}_{B},<, V\right\rangle$ satisfying $\Gamma$, where: $\mathcal{W}_{B}$ is the set of labels that appear in the branch $\mathbf{B}$; for each $x, y \in \mathcal{W}_{B}, x<y$ iff there exists $\Gamma_{i}$ in $\mathbf{B}$ such that $x<y \in \Gamma_{i}$; for each $x \in \mathcal{W}_{B}, V(x)=\left\{P \in A T M \mid\right.$ there is $\Gamma_{i}$ in $\mathbf{B}$ such that $x$ : $\left.P \in \Gamma_{i}\right\}$. We can easily prove that:

(i) by Theorem 3 , we have that $\mathcal{W}_{B}$ is finite;

(ii) $<$ is an irreflexive, transitive and modular relation on $\mathcal{W}_{B}$ satisfying the smoothness condition. Irreflexivity, transitivity and modularity are obvious, given Definition 4 and Lemma 2 above. Since $<$ is irreflexive and transitive, it can be easily shown that it is also acyclic. This property together with the finiteness of $\mathcal{W}_{B}$ entails that $<$ cannot have infinite descending chains. In turn this last property together with the transitivity of $<$ entails the smoothness condition.

(iii) We show that, for all formulas $F$ and for all $\Gamma_{i}$ in $\mathbf{B}$, (i) if $x: F \in \Gamma_{i}$ then $\mathcal{M}, x \mid=F$ and (ii) if $x: \neg F \in \Gamma_{i}$ then $\mathcal{M}, x \not \models F$. The proof is by induction on the complexity of the formulas. If $F \in A T M$ this immediately follows from definition of $V$. For the inductive step, due to space limitations, we only present the case of $F=A \sim B$. The other cases are similar and then left to the reader. Let $x: A \sim B \in \Gamma_{i}$. By Definition 4, we have that, for all $y$, there is $\Gamma_{j}$ in $\mathbf{B}$ such that either $y: \neg A \in \Gamma_{j}$ or $y: B \in \Gamma_{j}$ or $y: \neg \square \neg A \in \Gamma_{j}$. We show that for all $y \in M i n_{<}(A), \mathcal{M}, y \models B$. Let $y \in \operatorname{Min}_{<}(A)$. This entails that $\mathcal{M}, y \models A$, hence $y: \neg A \notin \Gamma_{j}$. Similarly, we can show that $y: \neg \square \neg A \notin \Gamma_{j}$. It follows that $y: B \in \Gamma_{j}$, and by inductive hypothesis $\mathcal{M}, y \models B$. (ii) If $x: \neg(A \sim B) \in \Gamma_{i}$, since $\mathbf{B}$ is saturated, there is a label $y$ in some $\Gamma_{j}$ such that $y: A \in \Gamma_{j}, y: \square \neg A \in \Gamma_{j}$, and $y: \neg B \in \Gamma_{j}$. By inductive hypothesis we can easily show that $\mathcal{M}, y=A, \mathcal{M}, y \models \square \neg A$, hence $y \in \operatorname{Min}_{<}(A)$, and $\mathcal{M}, y \mid \models B$, hence $\mathcal{M}, x \not \models A \sim B$.

\section{Termination of $\mathcal{T} R$ and Optimal Proof Search}

In this section, we refine $\mathcal{T} \mathbf{R}$ in order to ensure termination. Moreover, we describe an optimal decision procedure for $\mathbf{R}$ that allows to decide the satisfiability in $\mathbf{R}$ in nondeterministic polynomial time.

In general, non-termination in tableau calculi can be caused by two different reasons: 1. some rules copy their principal formula in the conclusion, so that they can be reapplied over the same formula without any control; 2 . dynamic rules can generate infinitely-many worlds, creating infinite branches.

As far as $\mathcal{T} \mathbf{R}$ is concerned, Theorem 3 excludes the second source of non termination (point 2). Concerning point 1, the above calculus $\mathcal{T} \mathbf{R}$ does not ensure a terminating proof search due to $\left(\sim^{+}\right)$, which can be applied without any control. We ensure the termination by putting some constraints on $\left(\sim^{+}\right)$in $\mathcal{T} \mathbf{R}$. It is easy to observe that it is useless to apply the rule on the same conditional formula more than once by using the 
same label $x$. Indeed, all formulas in the premise of $\left(\sim^{+}\right)$are kept in the conclusions, then we can assume, without loss of generality, that two applications of $\left(\sim^{+}\right)$on $x$ are consecutive. We observe that the second application is useless, since each of the conclusions has already been obtained after the first application, and can be removed. We prevent redundant applications of $\left(\mathrm{r}^{+}\right)$by keeping track of labels (worlds) in which a conditional $u: A \sim B$ has already been applied in the current branch. To this purpose, we add to each positive conditional a list of used labels; we restrict the application of $\left(\sim^{+}\right)$only to labels not occurring in the corresponding list. Notice that also the rule $(<)$ copies its principal formula $x<y$ in the conclusion; however, this rule will be applied only a finite number of times. This is a consequence of the side condition of the rule application and the fact that the number of labels in a tableau is finite (Theorem 3).

The terminating calculus $\mathcal{T} \mathbf{R}^{\mathbf{T}}$ is obtained by replacing the $\left(\sim^{+}\right)$rule in Figure 1 with the one presented in Figure 3.

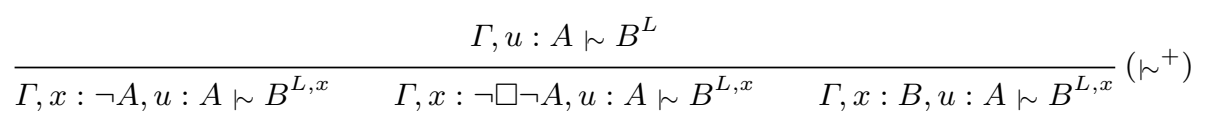

with $x \notin L$

Fig. 3. The rule $\left(\sim^{+}\right)$in the tableau system $\mathcal{T} \mathbf{R}^{\mathbf{T}}$.

Theorem 5 (Soundness and completeness of $\mathcal{T} \mathbf{R}^{\mathbf{T}}$ ). The calculus $\mathcal{T} \mathbf{R}^{\mathbf{T}}$ is sound and complete w.r.t. the semantics.

Theorem 6 (Termination of $\mathcal{T} \mathbf{R}^{\mathbf{T}}$ ). Let $\Gamma$ be a finite set of formulas, then any tableau generated by $\mathcal{T} \mathbf{R}^{\mathbf{T}}$ is finite.

Let $n$ be the size of the starting set $\Gamma$ of which we want to verify the satisfiability. The number of applications of the rules is proportional to the number of labels introduced in the tableau. In turn, this is $O\left(2^{n}\right)$ due to the interplay between the rules $\left(\sim^{+}\right)$ and $\left(\square^{-}\right)$. Hence, the complexity of the calculus $\mathcal{T} \mathbf{R}^{\mathbf{T}}$ is exponential in $n$.

In order to obtain a better complexity bound for validity in $\mathbf{R}$ we provide the following procedure. Intuitively, we do not apply $\left(\square^{-}\right)$to all negated boxed formulas, but only to formulas $y: \neg \square \neg A$ not already expanded, i.e. such that $z: A, z: \square \neg A$ do not belong to the current branch. As a result, we build a small model for the initial set of formulas in accordance with Theorem 1 . This is made possible by the modularity of $<$ in $\mathbf{R}$.

Let us define a nondeterministic procedure $\operatorname{CHECK}(\Gamma)$ to decide whether a given set of formulas $\Gamma$ is satisfiable. Let $\operatorname{EXPAND}(\Gamma)$ be a procedure that returns one saturated expansion of $\Gamma$ w.r.t. all static rules. In case of a branching rule, EXPAND nondeterministically selects (guesses) and applies one conclusion of the rule.

$\operatorname{CHECK}(\Gamma)$

1. $\Gamma \longleftarrow \operatorname{EXPAND}(\Gamma)$;

2. if $\Gamma$ contains an axiom then return UNSAT;

3. $\Gamma \longleftarrow$ result of applying $\left(\sim^{-}\right)$to each negated conditional in $\Gamma$;

4. $\Gamma \longleftarrow \operatorname{EXPAND}(\Gamma)$; 
5. if $\Gamma$ contains an axiom then return UNSAT;

while $\Gamma$ contains a $y: \neg \square \neg A$ not marked as CONSIDERED do

6. select $y: \neg \square \neg A \in \Gamma$ not already marked as CONSIDERED;

6a. if there is $z$ in $\Gamma$ such that $z: A \in \Gamma$ and $z: \square \neg A \in \Gamma$

then 6a'. add $z<y, \Gamma_{y \rightarrow z}^{M}$ to $\Gamma$;

else 6 a". $\Gamma \longleftarrow$ result of applying $\left(\square^{-}\right)$to $y: \neg \square \neg A$;

6b. mark $y: \neg \square \neg A$ as CONS IDERED;

7. $\Gamma \longleftarrow \operatorname{EXPAND}(\Gamma)$;

8. if $\Gamma$ contains an axiom then return UNSAT;

endWhile

9. return $\mathrm{SAT}$;

Observe that the addition of the set of formulas $z<y, \Gamma_{y \rightarrow z}^{M}$ in step 6a' could be omitted and it has been added mostly to enhance the understanding of the procedure. Indeed, the rule $(<)$, which is applied at each iteration to assure modularity, already takes care of adding such formulas. The procedure CHECK nondeterministically builds an open branch for $\Gamma$.

Theorem 7 (Soundness and completeness of the procedure). The above procedure is sound and complete w.r.t. the semantics.

Proof sketch. (Soundness). We prove that if the initial set of formulas $\Gamma$ is satisfiable, then the above procedure returns SAT. More precisely, we prove that each step of the procedure preserves the satisfiability of $\Gamma$. As far as EXPAND is concerned, notice that it only applies the static rules of $\mathcal{T} \mathbf{R}^{\mathbf{T}}$ and the soundness follows from the fact that these rules preserve satisfiability (see Theorems 2 and 5). Consider now step 6. Let $y: \neg \square \neg A$ the formula selected in this step. If $\left(\square^{-}\right)$is applied to $y: \neg \square \neg A$ (step 6a") we are done, since $\left(\square^{-}\right)$preserves satisfiability (see Theorems 2 and 5). If $\Gamma$ already contains $z: A, z: \square \neg A$, then step 6a' is executed, and the relation $z<y$ is added. In this case we reason as follows. Since $\Gamma$ is satisfiable, we have that there is a model $\mathcal{M}$ and a mapping $I$ such that $(1) \mathcal{M}, I(y) \models \neg \square \neg A$ and $(2) \mathcal{M}, I(z) \models A$ and $\mathcal{M}, I(z) \models \square \neg A$. We can observe that $I(z)<I(y)$ in $\mathcal{M}$. Indeed, by the truth condition of $\neg \square \neg A$ (see Definitions 2 and 3) and by the strong smoothness condition, we have that there exists $w$ such that $w<I(y)$ and $\mathcal{M}, w \models A, \square \neg A$. By modularity of $<$, either 1. $w<I(z)$ or $2 . I(z)<I(y)$. 1 is impossible, since otherwise we would have $\mathcal{M}, w \models \neg A$, which contradicts $\mathcal{M}, w \models A$. Hence, 2 holds. Therefore, we can conclude that step $6 \mathrm{a}^{\prime}$ preserves satisfiability.

(Completeness). It can be easily shown that in case the procedure above returns SAT, then the branch built is saturated (see Definition 4). Therefore, we can build a canonical model for the initial $\Gamma$, as done in the proof of Theorem 4.

Theorem 8 (Complexity of the CHECK procedure). By means of the procedure CHECK the satisfiability of a set of formulas of logic $\mathbf{R}$ can be decided in nondeterministic polynomial time.

Proof. Observe that the procedure generates at most $O(n)$ labels by applying the rule $\left(\sim^{-}\right)$(step 3) and that the while loop generates at most one new label for each $\neg \square \neg A$ formula. Indeed, the rule $\left(\square^{-}\right)$is applied to a labelled formula $y: \neg \square \neg A$ to generate a new world only if there is not a label $z$ such that $z: A \in \Gamma$ and $z: \square \neg A \in \Gamma$ are 
already on the branch. In essence, the procedure does not add a new minimal $A$-world on the branch if there is already one. As the number of different $\neg \square \neg A$ formulas is at most $O(n)$, then the while loop can add at most $O(n)$ new labels on the branch. Moreover, for each different label $x$, the expansion step can add at most $O(n)$ formulas $x: \neg \square \neg A$ on the branch, one for each positive conditional $A \sim B$ occurring in the set $\Gamma$. We can therefore conclude that the while loop can be executed at most $O\left(n^{2}\right)$ times.

As the number of generated labels is at most $O(n)$, by the subformula property, the number of labelled formulas on the branch is at most $O\left(n^{2}\right)$. Hence, the execution of step 6a has complexity $O\left(n^{2}\right)$. The execution of the nondeterministic procedure EXPAND has complexity $O\left(n^{2}\right)$, including a guess of size $O\left(n^{2}\right)$, whereas to verify if $\Gamma$ contains an axiom has complexity $O\left(n^{4}\right)$ (since it requires to check whether, for each labelled formula $x: P \in \Gamma$, the formula $x: \neg P$ is also in $\Gamma$, and $\Gamma$ contains at most $O\left(n^{2}\right)$ labelled formulas). We can therefore conclude that the execution of the CHECK procedure requires at most $O\left(n^{6}\right)$ steps.

By Theorem 8, the validity problem for $\mathbf{R}$ is in coNP. coNP-hardness is immediate, since $\mathbf{R}$ includes classical propositional logic. Thus, we can conclude that:

Theorem 9 (Complexity of $\mathbf{R}$ ). The problem of deciding the validity for rational logic $\mathbf{R}$ is coNP-complete.

\section{Conclusions and Future Work}

In this paper we have developed an analytic tableau calculus $\mathcal{T} \mathbf{R}$ for the rational logic $\mathbf{R}$. To the best of our knowledge, this is the first calculus for $\mathbf{R}$ directly based on preferential semantics. We have proved the termination of the calculus and provided a systematic procedure for deciding the satisfiability of a set of formulas in nondeterministic polynomial time. The paper is complementary to the work [15], where the other KLM systems are considered.

We briefly remark on some related work for deductive approaches to KLM logics.

Proof methods for the other KLM logics and for conditional logics related to them have been presented in [19-21]. Decidability of $\mathbf{P}$ and $\mathbf{R}$ has also been obtained by interpreting them into standard modal logics, as it is done by Boutilier [17]. However, Boutilier rejects the smoothness condition, which is essential in KLM framework. Furthermore, Boutilier gives a less natural and more complicated mapping into modal logic S4 and S4.3 for $\mathbf{P}$ and $\mathbf{R}$ respectively. Our logic and S4.3 are incomparable: finite-chain condition corresponding to the axiom $\mathrm{G}$ does not hold in S4.3, reflexivity and weak connectedness (holding in S4.3) do not hold in our logic. In [15] analytic tableaux calculi for $\mathbf{P}$ and $\mathbf{C L}$ are presented. These calculi are based on the same idea of using suitable modalities to interpret conditional assertions. In [15] authors show that the problem of deciding validity is co-NP complete for both logics $\mathbf{P}$ and $\mathbf{C L}$.

We plan to extend our calculi to the first order case. The starting point will be the analysis of first order rational logic by Friedman, Halpern and Koller in [4]. In subsequent research we also intend to investigate how to find models in the alternative semantics of $\mathbf{P}$ and $\mathbf{R}$ [3] of a set of conditional assertions by using our tableau methods. This could be a step in order to use our tableau procedures to uniformly implement a variety of default reasoning mechanisms built upon KLM logics $\mathbf{P}$ and $\mathbf{R}$ [5-8]. 


\section{References}

1. Kraus, S., Lehmann, D., Magidor, M.: Nonmonotonic reasoning, preferential models and cumulative logics. Artificial Intelligence 44(1-2) (1990) 167-207

2. Gardenförs, P.: Knowledge in Flux. MIT Press (1988)

3. Friedman, N., Halpern, J.Y.: Plausibility measures and default reasoning. Journal of the ACM 48(4) (2001) 648-685

4. Friedman, N., Halpern, J.Y., Koller, D.: First-order conditional logic for default reasoning revisited. ACM TOCL 1(2) (2000) 175-207

5. Benferhat, S., Dubois, D., Prade, H.: Nonmonotonic reasoning, conditional objects and possibility theory. Artificial Intelligence 92(1-2) (1997) 259-276

6. Benferhat, S., Saffiotti, A., Smets, P.: Belief functions and default reasoning. Artificial Intelligence 122(1-2) (2000) 1-69

7. Weydert, E.: System jlz - rational default reasoning by minimal ranking constructions. Journal of Applied Logic 1(3-4) (2003) 273-308

8. Pearl, J.: System z: A natural ordering of defaults with tractable applications to nonmonotonic reasoning. In: Proc. of the 3rd Conference on Theoretical Aspects of Reasoning about Knowledge, Morgan Kaufmann Publishers Inc. (1990) 121-135

9. Makinson, D.: Bridges from Classical to Nonmonotonic logic. London: King's College Publications. Series: Texts in Computing, vol 5 (2005)

10. Makinson, D.: Bridges between classical and nonmonotonic logic. Logic Journal of the IGPL 11(1) (2003) 69-96

11. Arieli, O., Avron, A.: General patterns for nonmonotonic reasoning: From basic entailments to plausible relations. Logic Journal of the IGPL 8(2) (2000) 119-148

12. Dubois, D., Fargier, H., Perny, P., Prade, H.: Qualitative decision theory: from savages axioms to nonmonotonic reasoning. Journal of the ACM 49(4) (2002) 455-495

13. Dubois, D., Fargier, H., Perny, P.: Qualitative decision theory with preference relations and comparative uncertainty: An axiomatic approach. Art. Int. 148(1-2) (2003) 219-260

14. Lehmann, D., Magidor, M.: What does a conditional knowledge base entail? Artificial Intelligence 55(1) (1992) 1-60

15. Giordano, L., Gliozzi, V., Olivetti, N., Pozzato, G.L.: Analytic Tableaux for KLM Preferential and Cumulative Logics. In: Proc. of LPAR 2005, LNAI 3835, Springer (2005) 666-681

16. Crocco, G., Lamarre, P.: On the connection between non-monotonic inference systems and conditional logics. In: Proc. of KR 92. (1992) 565-571

17. Boutilier, C.: Conditional logics of normality: a modal approach. Art. Int. 68(1) (1994) 87-154

18. Hughes, G., Cresswell, M.: A Companion to Modal Logic. Methuen (1984)

19. Artosi, A., Governatori, G., Rotolo, A.: Labelled tableaux for non-monotonic reasoning: Cumulative consequence relations. J. of Logic and Computation 12(6) (2002) 1027-1060

20. Giordano, L., Gliozzi, V., Olivetti, N., Schwind, C.: Tableau calculi for preference-based conditional logics. In: Proc. of TABLEAUX 2003, LNAI 2796, Springer (2003) 81-101

21. Giordano, L., Gliozzi, V., Olivetti, N., Schwind, C.: Extensions of tableau calculi for preference-based conditional logics. In: Proc. of M4M-4, Informatik-Bericht 194 (2005) 220-234 